\title{
Los centros de internamiento de extranjeros (CIE) en España: función, perfiles de la población internada y posibilidades de asistencia social en su interior*
}

[Artículos de investigación]

Laura Zanón Bayón-Torres ${ }^{* *}$

Recibido: o9 de septiembre de 2020

Aceptado: 12 de febrero de 2021

Citar como:

Zanón Bayón-Torres, L. (2021). Los centros de internamiento de extranjeros (CIE) en España: función, perfiles de la población internada y posibilidades de asistencia social en su interior. Campos en Ciencias Sociales, 9(1).

https://doi.org/10.15332/25006681.6926

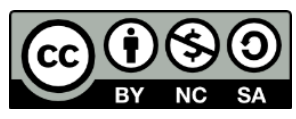

\section{Resumen}

En las dos últimas décadas, especialmente desde la crisis económica de 2008, la Unión Europea ha endurecido las políticas migratorias.

Actualmente, el control de los flujos migratorios se centra en tres objetivos: limitar la entrada de personas extranjeras, dificultar su

\footnotetext{
* Artículo de investigación elaborado en el desarrollo de la tesis doctoral para optar al grado de doctora en el Programa de Migraciones Internacionales y Cooperación al Desarrollo, impartido en el Instituto Universitario de Estudios sobre Migraciones (IUEM) de la Universidad Pontificia de Comillas de Madrid (España).

** Doctoranda en el Instituto Universitario de Estudios sobre Migraciones (IUEM) de la Universidad Pontificia de Comillas de Madrid (España). Correos electrónicos:

lauzanon@yahoo.es, Izanon@comillas.edu; ORCID: https://orcid.org/0000-0003-1573$\underline{6865}$
}

Campos en Ciencias Sociales

ISSN: 2339-3688 | e-ISSN: 2500-6681 | DOI: https://doi.org/10.15332/25394363

Vol. 9 N.० 1 | enero-junio de 2021 
regularización administrativa y facilitar su expulsión del territorio europeo. España, por sus peculiaridades geopolíticas, es uno de los países de entrada a Europa, tanto desde África como desde Latinoamérica, y, por tanto, presenta una actividad importante referente a internamientos y expulsiones de personas extranjeras, por lo que cuenta con varios centros de internamiento de extranjeros distribuidos por el país. Mediante una investigación empírica de corte cualitativo, el presente artículo identifica los principales retos que enfrentan los trabajadores sociales en su desempeño profesional en el centro de internamiento de extranjeros de Aluche. Para ello, se ha realizado una revisión bibliográfica y documental, y un análisis jurídico-normativo, en la que se reflexiona sobre algunas de las necesidades y características más relevantes de la población internada en el centro, y sobre cómo la propia estructura y el funcionamiento del centro limitan el quehacer profesional.

Palabras clave: trabajo social, asistencia social, política migratoria, personas migrantes, centros de internamiento de extranjeros.

\section{Foreign Internment Centers in Spain: Function, Profiles of the Interned Population, and Internal Social Assistance Possibilities}

\section{Abstract}

In the last two decades, there has been a tightening of immigration policies in the European Union, which has increased after the last economic crisis in 2008. Currently, the control of migratory flows focuses on three objectives: limiting the entry of foreigners, hindering their administrative regularization, and facilitating their expulsion from European territory. Spain, due to its geopolitical peculiarities, as it is the gateway to Europe from Africa and Latin America, presents an important activity in terms of detentions and expulsions, and has several detention centers for foreigners distributed throughout the country. Through qualitative empirical research, this article identifies the main challenges faced by social workers in their professional performance in the Aluche Internment Center. 
To that end, a bibliographic and documentary review and a legal-

normative analysis were carried out. Likewise, reflections on some of the most relevant needs and aspects related to the interned population as well as on how the structure and operation of the center limit the professional work are presented.

Keywords: social work, social assistance, immigration policies, migrants, foreign internment centers.

\section{Introducción al estudio: contextualización de los centros de internamiento de extranjeros en las políticas de control migratorio}

La Directiva 2008/115/CE del Parlamento Europeo y del Consejo (Directiva de Retorno), de 16 de diciembre de 2008, define los centros de internamiento de extranjeros (CIE) como centros públicos de privación de libertad, cuya finalidad es garantizar la expulsión de una persona extranjera en situación administrativa irregular de cualquier Estado miembro, mediante la custodia cautelar y preventiva. Los motivos por los que una persona puede ser internada en España son, principalmente, los siguientes: por ser parada e identificada en la calle por parte de la policía (práctica realizada en este país y conocida popularmente como "redadas racistas", ya que se llevan a cabo atendiendo únicamente al fenotipo de la persona, cuya realización se prohíbe tanto por el derecho español como por el derecho europeo), por ser detenida en frontera al tratar de acceder a territorio español por una vía no autorizada o, bien, por ser trasladada al CIE desde un establecimiento penitenciario.

La Ley 4/2000 de 11 de enero (LOEX), sobre derechos y libertades de los extranjeros en España y su integración social, distingue en su artículo 57 dos tipos de expulsiones: la de tipo administrativo y la penal. En el primer caso, la expulsión administrativa puede dictarse en el caso de que la persona haya entrado al país por una vía no autorizada (LOEX, 200o, art. 54), en el 
supuesto de migrantes que presenten un tiempo de estancia más o menos duradera en España y que se encuentren en situación administrativa irregular (LOEX, 2000, art. 53), de manera sobrevenida, o, bien, porque nunca hayan podido regularizar su situación.

En el caso de la expulsión penal (LOEX, 2000, art. 57.2), el artículo 89 de la Ley Orgánica 10/1995, de 23 de noviembre, del Código Penal recoge dos supuestos: que las personas sean expulsadas una vez cumplida la condena pena o que sean internadas en el CIE porque la expulsión supone la sustitución total o parcial de la condena de privación de libertad.

Independientemente del supuesto del que se trate, el artículo 15 de la Directiva de Retorno dictamina que el internamiento siempre debe ser autorizado por autoridades administrativas o judiciales. En España, la garantía judicial supera el mínimo previsto por dicha Directiva, ya que es condición sine qua non que un juez ordene el internamiento, previo informe de la Policía Nacional. Así pues, la Dirección General de la Policía, recogiendo lo establecido en la Directiva de Retorno y en la LOEX, publicó el 11 de julio de 2014 la Circular 6/2014, donde se establecen los criterios de internamiento en estos centros. En esta circular se recoge que, debido a la capacidad limitada de los centros de internamiento a la necesidad de constatar que la expulsión pueda ser ejecutada, cada caso debe investigarse individualmente, atendiendo a las circunstancias personales genéricas (como acreditar domicilio conocido, tener el pasaporte en vigor, presentar vínculos sociales o no apreciarse riesgo de fuga, entre otros) y específicas (por ejemplo, la edad, el estado de gestación, haber sido víctima de tortura o el estado de salud).

Sin embargo, las cifras constatan que el número de internamientos es muy superior al número de expulsiones efectivamente ejecutadas (Vargas, 2019; Gortázar Rotaeche, 2020). En esta línea, un informe publicado por el Servicio Jesuita a Migrantes (SJM) en 2018 señala que en esa fecha se 
documentó un considerable incremento de las entradas a España por las costas del sur del país, aumentando los internamientos de nacionales de Argelia y Marruecos. Esto tuvo como resultado que las dos terceras partes de las personas internadas ostentaran dichas nacionalidades. Por ello, el SJM (2018) afirma que "el internamiento se utilizó sobre todo como herramienta de las políticas de control de fronteras: el $92 \%$ de las personas argelinas y un $79 \%$ de las marroquíes fueron internadas por motivo de devolución por su entrada irregular en España” (p. 7). En opinión de esta entidad, debido a los acuerdos de repatriación firmados con estos dos países, se ha registrado un incremento en la ejecución de las expulsiones a lo largo de este último año (Martín, 2019), lo que consideran una discriminación por razón de origen. También en 2018, la Asociación Karibu Amigos del Pueblo Africano (2018) declaró que "la elevada cifra de personas liberadas dentro del colectivo subsahariano (93\%) confirma el absurdo del internamiento para personas de este origen. Es una privación de libertad injusta, ya que no está dirigida a facilitar la expulsión” (p. 49), que, además, infringe los principios establecidos en la Directiva de Retorno. Por tanto, siguiendo las informaciones publicadas por estas entidades, puede afirmarse que los acuerdos bilaterales de repatriación tienen un papel crucial en la decisión de internar a una persona por encima de las circunstancias individuales que presente cada caso. Es importante recordar en este punto que estos acuerdos son necesarios para el retorno, pero no están relacionados con la necesidad de privar de libertad, la cual, únicamente, se justifica si se impone como medida cautelar ante la existencia de indicios que permitan inferir que existe un riesgo de fuga (Directiva de Retorno, 2008, art. 15).

Así pues, los centros de internamiento se han convertido en una pieza clave de la política migratoria, cuyo funcionamiento fomenta la construcción de la Europa fortaleza y desestabiliza los procesos de integración social, 
condenando a la clandestinidad a las personas que pasan por ellos. Además, en el caso de personas condenadas, el internamiento en el CIE no computa como pena, constituyendo, muchas veces, un segundo internamiento que se produce entre la prisión y la ejecución de la expulsión. En conclusión, los CIE son:

[...] una pieza de una política migratoria de lucha contra la inmigración irregular asentada sobre la expulsión y la amenaza que representa, por lo que en la comprensión del internamiento de extranjeros no puede prescindirse de una mirada a la política a la que los CIE sirven como instrumento. (Martínez Escamilla, 2016, p. 3)

\section{Justificación, metodología y objetivos de la investigación}

El CIE de Madrid ha sido escogido como caso de estudio por presentar unas características especialmente relevantes. En primer lugar, como se detallará más adelante, en este centro se viene desarrollando la asistencia social por parte de Cruz Roja desde la firma de un convenio entre la entidad y el Ministerio del Interior en el año 2010, siendo el primer centro de todo el territorio nacional en el que comenzó a ofrecerse una asistencia social profesionalizada desarrollada por profesionales del trabajo social. Por otro lado, su ubicación y la red de recursos asistenciales, y de otra naturaleza, también, de la que dispone la Comunidad de Madrid convierten al CIE de Aluche es un dispositivo donde se internan a personas extranjeras de todos los lugares de España, concentrándose en su interior una amplia variedad de situaciones y circunstancias personales y sociofamiliares.

Para llevar a cabo este estudio de caso, se ha utilizado una metodología de carácter cualitativo basado en el análisis documental, bibliográfico y de fuentes jurídicas.

Como se ha comentado, los centros de internamiento de extranjeros fueron concebidos para facilitar la ejecución de las expulsiones del territorio 
nacional de personas extranjeras extracomunitarias, que han accedido al país por una vía no autorizada o bien presentan periodos más o menos largos de estancia irregular en España. En este último caso, pueden ser migrantes que se han encontrado con una situación de irregularidad administrativa sobrevenida o personas que no han logrado regularizarse en el tiempo que han permanecido en el país. Sin embargo, el internamiento únicamente debería producirse para que una expulsión prevista no se vea frustrada. Por tanto, aunque no todas las personas migrantes han sido o serán internadas en un CIE, resulta fundamental para el ejercicio del trabajo social saber qué es y cómo afecta al colectivo con el que se interviene temer o sufrir un internamiento en este tipo de centros. Por ello, el objeto de esta investigación es la identificación de los retos profesionales en este contexto, habiéndose establecido para ello dos objetivos generales:

1. Analizar la normativa vigente que rige el desempeño de la profesión en el CIE de Aluche.

2. Identificar las problemáticas más relevantes de la población atendida.

\section{Marco jurídico de la asistencia social en el CIE de Aluche}

La naturaleza jurídica del CIE y su funcionamiento, incluida la asistencia social que debe prestarse en él, se regulan en diferentes normas. Su redacción, publicación y aplicación no ha estado exenta de polémica y críticas procedentes, principalmente, de organismos, entidades y profesionales defensores de los derechos humanos o de la oficina del Defensor del Pueblo.

Las críticas a los CIE son numerosas ya que, de facto, en palabras de García España (2017), constituyen "una privación del derecho fundamental a la libertad sin que medie un comportamiento delictivo. Consiste, en última instancia, en una prolongación de las 72 horas permitidas legalmente de detención policial con autorización judicial” (p. 2). Así, pues, su regulación y

Campos en Ciencias Sociales

ISSN: 2339-3688 | e-ISSN: 2500-6681 | DOI: https://doi.org/10.15332/25394363

Vol. 9 N. ${ }^{\circ} 1$ | enero-junio de 2021 
funcionamiento son dos de los grandes problemas que presenta esta institución, al considerarse que la normativa no es lo suficientemente garantista y que su aplicación difiere de unos centros de internamiento a otros.

En España, la regulación de los CIE comienza con la Ley Orgánica 7/1985, de 1 de julio, sobre derechos y libertades de los extranjeros en España. En su artículo 26, se estableció que el internamiento era concebido como una medida cautelar acordada por un juez de instrucción para la sustanciación o ejecución de una orden de expulsión, esta medida no podía exceder de cuarenta días el periodo de internamiento y se debía llevar a cabo en "centros de detención o en locales no penitenciarios". Ese mismo año, fue presentado un recurso de inconstitucionalidad por parte la oficina del Defensor del Pueblo, fundamentado, entre otras cuestiones, en que pudiera decretarse una privación de libertad por la comisión de una falta administrativa, contrariamente a lo que promulga el artículo 25.3 de la Constitución española ${ }^{1}$. No obstante, la Sentencia 115/1987 de 7 de julio del Tribunal Constitucional sentenció que, bajo un mandato judicial y cumpliéndose determinados requisitos, sí que podría llevarse a cabo un internamiento. Sin embargo, no fue hasta el año 2003 cuando aspectos fundamentales como el funcionamiento interno de los CIE, la asistencia social, los derechos y deberes de las personas internadas o la información que se les debe ofrece se regularon en una norma con rango de ley², mediante desarrollo normativo de la LOEX.

\footnotetext{
${ }^{1}$ De acuerdo con el artículo citado en el texto, la administración civil no podrá imponer sanciones que, directa o subsidiariamente, impliquen privación de libertad.

2 Fue en ese año cuando se aprobó la reforma de la Ley Orgánica 4/2000, del 11 de enero, sobre derechos y libertades de los extranjeros en España y su integración social, llevada a cabo por la Ley Orgánica 14/2003, del 20 de noviembre, quedando recogidos, en los artículos 62 bis a 62 sexies y en el artículo 258 del Reglamento de la Ley de Extranjería, aspectos relacionados con los CIE.
} 
En el año 2008, la Directiva de Retorno estableció en seis meses el plazo máximo del internamiento, pudiéndose prorrogar a dieciocho en determinados supuestos. Este hecho, el retorno de menores no acompañados o la prohibición de entrada fueron algunas de las cuestiones más controvertidas de la norma (Gortázar Rotaeche, 2009). No obstante, actualmente el ordenamiento jurídico no permite un internamiento superior a sesenta días, por lo que, en este caso, la normativa española es más garantista que la europea, a pesar de que el plazo fue ampliado de cuarenta días a sesenta. Sin embargo, el Consejo de Europa mediante una posición parcial de negociación acerca de la nueva Directiva de Retorno ha acordado aumentar el número de retornos efectivos ejecutados (Gortázar Rotaeche, 2020), por lo que el plazo de internamiento máximo podría verse incrementado.

Durante décadas, las entidades defensoras de los derechos humanos defendieron la necesidad de aprobar un reglamento específico para regular el funcionamiento de los CIE y garantizar adecuadamente los derechos de las personas internadas en ellos. A pesar de la aprobación del Real Decreto 162/2014, de 14 de marzo, por el que se aprueba el reglamento de funcionamiento y régimen interior de los centros de internamiento de extranjeros (RCIE), este no satisfizo las expectativas de los profesionales ni de las ONG, por considerarlo insuficiente, debido, entre otros motivos, al rechazo de las enmiendas presentadas (Público, 2008).

El RCIE establece en su artículo 1 que estos centros son:

[...] establecimientos públicos de carácter no penitenciario, dependientes del Ministerio del Interior, destinados a la custodia preventiva y cautelar de extranjeros para garantizar su expulsión, devolución o regreso por las causas y en los términos previstos en la legislación de extranjería, y de los extranjeros que, habiéndoseles sustituido la pena privativa de libertad por la medida de expulsión, el juez o tribunal competente así lo acuerde en

Campos en Ciencias Sociales

ISSN: 2339-3688 | e-ISSN: 2500-6681 | DOI: https://doi.org/10.15332/25394363

Vol. 9 N.o 1 | enero-junio de 2021 
aplicación de lo dispuesto por el artículo 89.6 del Código Penal. (Real Decreto 162/2014, art. 1)

Continúa este artículo señalando que "El ingreso y estancia en los centros tendrá únicamente finalidad preventiva cautelar, y estará orientado a garantizar la presencia del extranjero durante sustanciación del expediente administrativo y la ejecución de la medida de expulsión, devolución o regreso" (Art. 1. RCIE). Es decir, el reglamento determina que la naturaleza jurídica de los CIE es ser lugares donde se lleva a cabo un internamiento como medida cautelar que garantice la presencia de la persona que va a ser expulsada, con el fin de que no pueda frustrarse la deportación.

Un año después de la aprobación del RCIE, el Tribunal Supremo suspendió cuatro de sus artículos por considerarlos contrarios a la LOEX y a la Directiva de Retorno. En ellos, se permitían los cacheos con desnudo integral y los ingresos sucesivos de la misma persona y no quedaba garantizado el derecho a la intimidad de las familias. Sin embargo, el Tribunal no aceptó la impugnación de dos artículos fundamentales como es la posibilidad de que la policía porte armas dentro del recinto o la utilización de los CIE como centros de acogida temporal (Eldiario.es, 2015), uso que contraviene el propio RCIE. A pesar de todo, la necesidad de concreción y regulación del funcionamiento de los centros de internamiento se trató de satisfacer mediante la publicación dicho reglamento, derogándose la Orden del Ministerio de la Presidencia de 22 de febrero de 1999 sobre normas de funcionamiento y régimen interior de los centros de internamiento de extranjeros, vigente hasta ese momento, y trasponiéndose algunas de las indicaciones recogidas en la Directiva de Retorno.

Uno de los aspectos claves que se regulan en el RCIE es la atención social, además de las competencias en materia de dirección y gestión y de asistencia médica, cultural y jurídica. Como se recoge su artículo 8.2., las 
funciones de dirección y seguridad del centro se desempeñarán por miembros del Cuerpo Nacional de Policía, mientras que los servicios asistenciales, logísticos y administrativos serán desarrollados por empleados de las administraciones públicas. Concretamente, la atención social se considera un derecho de los internos (RCIE, art.16.2.) y está supeditada al control y supervisión por parte de la junta y de la dirección (RCIE, art.15), cargo desempeñado por la Policía Nacional.

En el mismo artículo 15 del RCIE se dispone que profesionales del trabajo social se encargarán de la atención social y cultural y deberán someter al análisis de la junta de coordinación los planes y proyectos de actuación. Según el artículo 10 del RCIE, dicha junta actuará como un órgano colegiado, harán parte de esta el director, el administrador, los responsables de la unidad de seguridad, el secretario de dirección y los responsables de la asistencia sanitaria y social. Es decir, según la norma que regula el funcionamiento interior del CIE, el planteamiento de la intervención social es analizado y autorizado por un órgano colegiado en el que los profesionales del trabajo social apenas se encuentran representación, mientras que la Policía Nacional ocupa casi la totalidad de los cargos de la junta. Este hecho demuestra que la intervención social está supeditada a la gestión policial y securitaria del centro.

Por otro lado, el art. 15.2 del RCIE contempla la posibilidad de que se suscriban acuerdos, convenios o contratos con entidades públicas o privadas y con las ONG que se consideren, para que estas organizaciones puedan prestar el servicio de atención social. Así, se señala que los profesionales que trabajen en el servicio de asistencia social deberán tener conocimientos en materia de extranjería, derechos humanos, género, protección internacional y mediación intercultural. Asimismo, se recoge que "la asistencia social y cultural se orientará fundamentalmente a la resolución de los problemas 
surgidos a los extranjeros internados y, en su caso, a sus familias" (RCIE, art. 15.3).

Para cumplir con el precepto del art. 15.2. del RCIE, en 2010 el Ministerio del Interior y la Cruz Roja acordaron, mediante el Real Decreto 167/2010, de 19 de febrero, por el que se regula la concesión de una subvención directa a favor de Cruz Roja Española para el desarrollo de programas de asistencia integral en el centro de internamiento de extranjeros de Madrid durante el ejercicio 2010 (Real Decreto 167/2010), que esta entidad desarrollara los programas de atención social en el CIE de Aluche. Actualmente, este convenio se ha hecho extensivo a todos los centros de internamiento del país. Según se explica en el documento, la subvención se le otorga a Cruz Roja, ya que reúne los requisitos suficientes, y se determina que resulta improcedente la realización de "una convocatoria pública para el otorgamiento de la subvención, máxime dada la urgencia con que es necesario reforzar los medios disponibles para la atención a los inmigrantes que se encuentran en el Centro de Internamiento de Extranjeros de Madrid" (Real Decreto 167/2010).

Por tanto, el convenio dicta en su artículo 1 que le corresponde a esta entidad desarrollar la intervención con las personas internas; el contacto con sus familias, tanto en España como en sus países de origen; la interpretación de las lenguas y las relaciones con el exterior, la tramitación de los documentos; la mediación social entre internos y personal del centro y la planificación y desarrollo de las actividades. Por su parte, en el artículo 7 del Real Decreto 167/2010, el Ministerio queda obligado a facilitar espacios adecuados donde el personal de la Cruz Roja pueda entrevistar a las personas internadas, con la privacidad y confidencialidad necesarias.

En el año 2017, tras sucesivas visitas a diferentes centros de internamiento, el Defensor del Pueblo realizó unas recomendaciones en relación con la asistencia jurídica, social y cultural. En referencia a las dos últimas, 
recalcaba la inexistencia de proyectos elaborados por profesionales del trabajo social y aprobados por la Junta para la intervención social, la falta de organización de actividades de ocio suficientes, la carencia de un servicio de traducción eficaz y la falta de ropa, calzado y útiles de higiene para las personas internadas. En sus visitas, constató que estas carencias diferían de unos centros a otros, como en el caso del abastecimiento de indumentaria y de productos de higiene personal. Asimismo, consideró que esta cuestión no quedaba recogida adecuadamente en el RCIE y añadió que, en materia asistencial, jurídica y cultural, no se habían acometido las reformas anunciadas en la citada norma. Uno de los motivos alegados que podría explicar las diferencias entre los distintos CIE que hay repartidos por el país radica en la dependencia jerárquica de la plantilla respecto a la figura del director en cada centro de internamiento (Defensor del Pueblo, 2017).

En relación con la población atendida y pesar de la vulnerabilidad que pueda asociarse a determinados colectivos, la normativa y, concretamente, el RCIE no prohíben su internamiento, sino que recomiendan la aplicación del "principio de proporcionalidad en los medios utilizados y objetivos perseguidos, el de intervención menos restrictiva y el de atención especializada a personas vulnerables" (RCIE, art. 1). Asimismo, en dicho artículo, se consideran personas vulnerables a "menores, personas discapacitadas, ancianos, mujeres embarazadas, padres solos con hijos menores y personas que hayan padecido tortura, violación u otras formas graves de violencia psicológica, física o sexual” (RCIE, Art.1). En el caso de los menores, su internamiento no está prohibido, pero sí condicionado a la autorización por parte del Ministerio Fiscal y a que el centro disponga de módulos adecuados para garantizar la intimidad de la unidad familiar (RCIE, art. 16.2.k.), circunstancia que actualmente no queda garantizada en este centro. En el caso de que sean internadas mujeres embarazadas, el art. 
16.1.f. del RCIE establece como derecho que puedan contar con una asistencia médica especial.

Es decir, como hemos visto a lo largo de estas líneas, además de la situación administrativa irregular y del sufrimiento que conlleva la privación de libertad, las personas internadas pueden presentar otras situaciones de vulnerabilidad que se ven agravadas por la detención y el internamiento y a las que debe dar respuesta el equipo que realiza la asistencia social, con una importante escasez de medios y limitación de funciones.

\section{Discusión y resultados relativos del estudio a los retos de la asistencia social en el CIE de Aluche}

Para comprender adecuadamente los retos del trabajo social en este contexto, es necesario insistir en que el internamiento y la expulsión no deberían materializarse cuando la persona pueda demostrar arraigo ni tampoco si el ingreso en un centro de estas características supone un riesgo para su integridad. Como se ha visto, el ejercicio de la profesión en este contexto no está exento de contradicciones y dificultades, las cuales se agudizan por la dureza de las situaciones sociales y personales del colectivo atendido, así como por la arquitectura y estructura del recinto.

El CIE de Madrid es uno de los siete centros de internamiento en España que existen en la actualidad. Su ubicación y los servicios con los que cuenta la ciudad determinan algunas de sus características. Está situado dentro del casco urbano de la capital, en la zona hospitalaria de la antigua cárcel de Carabanchel, presentando importantes reminiscencias carcelarias. Además, comparte recinto con la Comisaría de Policía Nacional de Latina, lugar en el que se gestionan un gran número de solicitudes de asilo. Es decir, en el mismo edificio donde se encuentra uno de los mayores centros de deportación del país, se ubica una Comisaría de la Policía Nacional encargada de abrir los expedientes de expulsión y de atender un elevado 
número de solicitudes de asilo. Las comunicaciones terrestres y aeroportuarias, así como los recursos que integran la red de atención social, son otros de los aspectos para tener en cuenta para comprender el funcionamiento de este CIE. Madrid tiene uno de los aeropuertos de mayor tráfico aéreo de Europa y se encuentra bien comunicada por vía terrestre con todos los puntos del país. Además, cuenta con una importante red de recursos atención (El País, 2019), por lo que recibe muchas personas procedentes, tanto de los CIE del sur como de otras comunidades autónomas donde no hay centros de internamiento.

Debido a la variedad de circunstancias que puede presentar la población atendida, resulta imposible hacer una enumeración exhaustiva de todas las necesidades expresadas o sentidas por parte de las personas internadas en el CIE; aunque, sí pueden destacarse algunas de las más habituales y acuciantes, derivadas de la situación personal y social o provocadas por el funcionamiento del propio centro, que deben ser gestionadas por profesionales del trabajo social.

En primer lugar, hay que destacar que resulta muy complicado planificar la intervención debido al desconocimiento, por parte de profesionales y de personas internadas, de la fecha de ejecución de la expulsión y, por tanto, del tiempo de internamiento efectivo (más allá del límite de sesenta días establecidos por ley) (El diario.es, 2015). La gravedad de este hecho se ve acrecentada debido a la variedad de colectivos que son atendidos en este CIE y a las delicadas circunstancias personales y vitales que presentan en la mayoría de los casos.

Así, una de las situaciones más preocupantes que se han detectado es la presencia de menores de edad (Defensor del Pueblo, 2019; Karibu, 2018; SJM, 2018; SOS Racismo, 2018), en su mayoría interceptados al cruzar la frontera como infancia no acompañada. El Comité de Derechos del Niño le ha llamado la atención a España en varias ocasiones por asignar la mayoría 
de edad a menores que aseguraban y, en ocasiones, acreditaban con documentos ser menores de edad (La Vanguardia, 2019). Pese a la gravedad de la situación, esta no es la única manera en la que la infancia es afectada por el CIE, sino que se ha comprobado que pueden producirse situaciones de desprotección cuando sus progenitores son detenidos por controles de identificación (redadas racistas), como ocurre con muchas mujeres que tienen menores a su cargo (Martínez Escamilla, 2013; SOS Racismo,2018).

Otra de las problemáticas más acuciantes es la presencia de posibles víctimas de trata (SOS Racismo, 2018). El Defensor del Pueblo (2019) recalca que este CIE presenta un cumplimiento parcial o incompleto sobre información referida a víctimas de trata, víctimas de violencia de género y otras formas de violencia, produciéndose deportaciones de personas que presentan indicios de ser víctimas de trata.

Por otro lado, la presencia de personas que padecen algún tipo de enfermedad y la atención médica dispensada son algunas de las quejas más repetidas en los informes de las ONG. El SJM (2018) denuncia una insuficiente dispensación de medicamentos y una práctica no sistemática de pruebas de detección de enfermedades infectocontagiosas. Por su parte, Karibu (2018) reivindica la necesidad de ofrecer atención psicológica a las personas internadas que así lo precisen. Según el Defensor del Pueblo (2019), no hay una adecuada atención médica, al carecer de un servicio de 24 horas, de una habitación de ingresos en enfermería y de remisión de parte de lesiones al juzgado por parte del servicio médico. Asimismo, también se registran, en el citado informe, carencias respecto al ocio, la ropa y el calzado suficientes, la asistencia social y cultural y el servicio de interpretación.

Otro aspecto alarmante es el conjunto de condiciones en las que se producen las solicitudes de protección internacional, detectándose una aplicación inadecuada de la normativa procedimental por parte de la Policía 
(Karibu, 2018; SJM, 2018). Esta circunstancia es extremadamente preocupante debido a que se ha incrementado la cantidad de solicitudes presentadas en el CIE procedentes de personas interceptadas en la frontera, dado que es uno de los centros donde primero son trasladadas al llegar a las costas españolas (SJM, 2014).

Por último, a pesar de que la normativa europea no permite que se internen extranjeros comunitarios, en la Guía práctica de asistencia jurídica en centros de internamiento de extranjeros, publicada por la Fundación Fernando Pombo en 2018, se expone que, en "el internamiento [que se haya] producido como consecuencia de una expulsión acordada en un procedimiento de carácter penal" (p. 15), sí que se produce el ingreso de un ciudadano comunitario en un CIE, circunstancia propiciada por la reforma en 2015 del artículo 89 del Código Penal. Sobre esta cuestión se ha pronunciado el Defensor del Pueblo (2019) considerando que la separación interior de las personas condenadas de las quienes no lo están, no se realiza adecuadamente.

Es decir, con los datos aportados, puede concluirse que la mayoría de las personas internadas, independientemente del tipo de expulsión que presenten, se encuentran en una situación de gran vulnerabilidad que no desaparecerá tras la finalización del internamiento, sino que lo más probable es que se agrave.

\section{Conclusiones}

El análisis de la realidad descrita en el artículo permite realizar algunas reflexiones en relación con el funcionamiento y "uso de facto" del CIE y sus implicaciones en el desempeño profesional de los profesionales del trabajo social. Aun así, las conclusiones que podemos aportar son muy limitadas, ya que en España todavía se carece de estudios científicos que hayan 
investigado cómo se produce el ejercicio del trabajo social en los centros de internamiento de extranjeros del país.

En primer lugar, se concluye que los centros de internamiento de extranjeros son espacios de la política de extranjería española y europea usados, a pesar de que fueron concebidos como una medida cautelar para asegurar la ejecución de la expulsión de una persona extranjera extracomunitaria que no contara con un documento válido que acreditara su estancia legal en territorio español, de forma inadecuada, frente a lo establecido en la normativa. Aunque la Directiva de Retorno no prohíbe el internamiento de personas que presenten algún indicio de vulnerabilidad, sí que recuerda la necesidad de que la privación de libertad sea impuesta solo si no existe otra medida alternativa contemplada en la legislación y, en todo caso, si se prevé que la expulsión puede ser efectivamente ejecutada, aspecto que no es respetado en base al elevado número de internamientos respecto al número de expulsiones finalmente llevadas a término. Por tanto, un elevado porcentaje de las personas internadas serán liberadas sin que se haya ejecutado su expulsión, pero habrán visto agravada su situación de vulnerabilidad debido a la privación de libertad sufrida.

Por tanto, el internamiento se autoriza sin cumplir de manera rigurosa los criterios establecidos por las normas vigentes, provocando, entre otras situaciones, vulneraciones de derechos humanos y desamparo de menores. Asimismo, a pesar de que la privación de la libertad tiene una duración máxima de sesenta días, esta obstaculiza e interrumpe procesos de integración socioeconómica y proyectos vitales y familiares, siendo un elemento disruptivo y amenazante para las personas más vulnerables que se encuentran en un proceso de integración en un país que no es el suyo de origen. A estas personas les resulta imposible gestionar con los medios humanos y materiales actuales del CIE de Aluche. Esto supone que la asistencia social se realice en el ámbito de la urgencia, sin poder abordar de 
manera adecuada las situaciones y sin tener capacidad para ofrecer acompañamiento a las personas que son liberadas. Ellas finalizan su internamiento con una expulsión, condenados a vivir en España de manera clandestina o, en el mejor de los casos, siendo derivados a programas de ayuda humanitaria que cuentan con recursos limitados y, la mayoría de las veces, con posibilidades de estancia inferiores a un año.

A pesar de que la aprobación del RCIE supuso un importante paso para la regulación del funcionamiento de estos centros, no ha impedido que sigan existiendo vulneraciones de derechos humanos ni tampoco ha logrado armonizar el funcionamiento de todos los CIE del país. La asistencia social es uno de los aspectos que se consideran insuficientemente desarrollados y protegidos. Depender jerárquicamente de la policía convierte al ejercicio de la profesión y al desarrollo de la intervención en aspectos secundarios frente a la custodia y retención de las personas internadas.

Por tanto, puede afirmarse que existen elementos que dificultan el ejercicio del trabajo social dentro de estos centros. Varios de ellos están relacionados con depender de forma jerárquica y orgánica de la policía, la cual podría estar limitando el quehacer profesional con base en criterios ajenos a esta disciplina. Algunos de estos criterios son la retención y custodia, ejes fundamentales del funcionamiento de estos centros, pero no de la asistencia social, así como el desconocimiento del plazo de internamiento de una persona o la diversidad de situaciones vitales y personales de la población retenida.

Es decir, el desempeño del trabajo social se encuentra envuelto en contradicciones, dilemas y dificultades que los equipos deben enfrentar en su día a día. Todo ello supone que, como en otros ámbitos, el ejercicio del trabajo social en este contexto se lleve a cabo desde el asistencialismo y la urgencia; probablemente, a costa de un gran desgaste emocional y de un escaso reconocimiento social del trabajo realizado. 


\section{Referencias}

Arango, J. (2005). Dificultades y dilemas de la política de inmigración. Arbor, 181(713), 1725. http://dx.doi.org/10.3989/arbor.2005.i713.439

Cabrera Cabrera, P. J. (2007). Exclusión social: contextos para un concepto. RTS: Revista de Treball Social, (180), 9-21.

Carbajosa, A. (2017, 23 de marzo). La vida después del CIE. El País. https://elpais.com/politica/2017/02/02/actualidad/1486027852_297434.html

CEAR. (2014). Diccionario de asilo. CEAR. https://diccionario.cear-euskadi.org/

Davis, A. (1981). Mujeres, raza y clase (3. ${ }^{\mathrm{a}}$ ed.). Ediciones Akal.

Defensor del Pueblo. (2017). Recomendación de 14 de diciembre. Centro de internamiento de extranjeros. Asistencia social, cultural y jurídica. https://www.defensordelpueblo.es/resoluciones/asistencia-social-cultural-yjuridica-en-los-centros-de-internamiento-de-extranjeros/

Defensor del Pueblo. (2019). Informe anual 2018. Mecanismo nacional de prevención. https://www.defensordelpueblo.es/wpcontent/uploads/2019/o9/Informe 2018 MNP.pdf

El País. (2005, 20 de junio). El nuevo centro de internamiento de extranjeros abre esta semana. El País.

https://elpais.com/diario/2005/06/20/madrid/1119266662 850215.html

Eldiario.es. (2015, 27 de julio)Un juez reitera a la Policía que debe notificar a los internos del CIE de Madrid la fecha y detalles de su expulsión. (27 de julio de 2015). Eldiario.es. https://www.eldiario.es/desalambre/Policia-notificar-CIE-Madridexpulsion o 413659174.html

Fundación Fernando Pombo. (2018). Guía práctica de asistencia jurídica en Centros de Internamiento de Extranjeros. Fundación Fernando Pombo.

García España, E. (2017). Centros de Internamiento de Extranjeros: Motivos para su desaparición. Boletín Criminológico, (23), 1-6. http://dx.doi.org/10.24310/Boletincriminologico.2017.v23io.3878

García España, E. (2018). El arraigo de presos extranjeros: más allá de un criterio limitador de la expulsión. Migraciones. Publicación del Instituto Universitario de Estudios sobre Migraciones, (44), 119-144. https://doi.org/10.14422/mig.i44.y2018.005 
González Sánchez, I. (2016). La penalización de los migrantes: irregularidad y cárcel en la construcción del estado neoliberal. Migraciones. Publicación del Instituto Universitario de Estudios sobre Migraciones, (39), 123-147. https://doi.org/10.14422/mig.i39.y2016.005

Gortázar Rotaeche, C. (1028, 18 de septiembre) Sobre vergüenza y dignidad en el retorno de los inmigrantes en situación irregular. Comillas.

https://blogs.comillas.edu/buildingbridges/2018/og/18/sobre-verguenza-ydignidad-en-el-retorno-de-los-inmigrantes-en-situacion-irregular-por-cristinagortazar-rotaeche/

Gortázar Rotaeche, C. (2009) Las nuevas normativas europeas sobre inmigración: perspectivas y riesgos. Especial mención a la llamada Directiva “de retorno". Corintios XIII: Revista de teología y pastoral de la caridad, (131), 29-41.

Gortázar Rotaeche, C. (2016). Conferencia internacional: "el arraigo frente a la expulsión: la protección de los extranjeros en Europa”. Migraciones. Publicación del Instituto Universitario de Estudios sobre Migraciones, (5), 249-257. https://revistas.comillas.edu/index.php/revistamigraciones/article/view/4477

Gortázar Rotaeche, C. (2020). Return Decisions and Domestic Judicial Practices: Is Spain Different? En M. Moraru, G. Cornelisse y P. Bruycker (Coords.), Law and Judicial Dialogue on the Return of Irregular Migrants from the European Union (pp. 1-23). Hart Publishing Books.

Karibu. (2018). Subsaharianos en el CIE de Aluche. Encerrados tras un muro de vergüenzas. https://www.asociacionkaribu.org/doc/KARIBU-informeCIE-2018.pdf

La Vanguardia (2019, 23 de octubre). El periplo de dos niños extranjeros no acompañados que al llegar a España no fueron reconocidos como menores. La Vanguardia. https://www.lavanguardia.com/vida/20191023/471157281675/el-periplo-de-dosninos-extranjeros-no-acompanados-que-al-llegar-a-espana-no-fueron-reconocidoscomo-menores.html

Manzanedo, C. (2013). Centros de Internamiento de Extranjeros en España: nada cambia. Migraciones Forzadas, (44), 18-19. https://www.fmreview.org/es/detencion/manzanedo

Martín, M. (2019, 6 de junio). Interior bate récords de expulsiones desde los CIE. El País. https://elpais.com/politica/2019/06/02/actualidad/1559507111 004291.html 
Martínez Escamilla, M. (2014). Expulsión e internamiento de extranjeros ¿Quiénes están en los CIE? En Reig Fabado, I. (Coord.), Libertad de circulación, asilo y refugio en la Unión Europea (pp. 347-376). Tirant Lo Blanch.

Martínez Escamilla, M. (2016). Centros de Internamiento para Extranjeros. Estado de la cuestión y perspectivas de futuro. Revista Electrónica de Ciencia Penal y Criminología, (18-23), 1-38. http://criminet.ugr.es/recpc/18/recpc18-23.pdf

Martínez Escamilla, M. (2013). Mujeres en el CIE. Género, inmigración e internamiento. Informe técnico. Tercera prensa; Hirugarren Prentsa S. L.

Nicolás Lizama, M. (2018). Los informes de arraigo: un análisis desde la perspectiva de los Servicios Sociales. Cuadernos De Trabajo Social, 31(1), 85-93. https://doi.org/10.5209/CUTS.56002

Público. (2018, 19 de junio). La Eurocámara aprueba la directiva de la vergüenza. Público. https://www.publico.es/actualidad/eurocamara-aprueba-directiva-vergueenza.html

Releño Pastor, E. (2015). Preocupaciones del Defensor del Pueblo en relación con la detención, internamiento y expulsión de ciudadanos extranjeros. En Martínez Escamilla, M. (Coord.), Detención, internamiento y expulsión administrativa de personas extranjeras (pp. 81-135). Universidad Complutense de Madrid. http://eprints.ucm.es/34492

Sánchez, G. (2014, 14 de marzo). El Gobierno subcontratará a ONG para asistencia social y sanitaria en los CIE. Eldiario.es. https://www.eldiario.es/desalambre/Gobiernosubcontratara-ONG-asistencia-CIE o $238676690 . h t m l$

Sánchez, G. (2014, 20 de octubre). Bruselas dice que España "no aplica" la norma europea de expulsión de inmigrantes en Ceuta y Melilla. Eldiario.es. https://www.eldiario.es/desalambre/Comision-Europea-Espana-normativainmigrantes o 315668604.html

Sánchez, G. (2015, 27 de enero). El Supremo suspende parte del reglamento de los CIE por incumplir la normativa europea y española. Eldiario.es.

https://www.eldiario.es/desalambre/Supremo-reglamento-CIE-LeyExtranjeria o $350316044 \cdot \mathrm{html}$

Servicio Jesuita a Migrantes. (2013). Informe CIE 2013: criminalizados, internados, expulsados. https://sjme.org/gallery/informe-cie-2013-criminalizados-internadosexpulsados/ 
Servicio Jesuita a Migrantes. (2014). Informe CIE 2014. Expulsiones exprés. https://sjme.org/gallery/informe-cie-2014-expulsiones-expres/

Servicio Jesuita a Migrantes. (2015). Vulnerables vulnerabilizados. Informe CIE 2015. https://sjme.org/gallery/vulnerables-vulnerabilizados-informe-cie-2015/

Servicio Jesuita a Migrantes. (2016). 25, 66 Media diaria de repatriaciones forzadas. Informe CIE 2016. https://sjme.org/gallery/lanzamiento-2566-informe-sjmcentros-internamiento-extranjeros-2016/

Servicio Jesuita a Migrantes. (2017). Informe CIE 2017 de SJM: Sufrimiento Inútil. https://sjme.org/gallery/informe-cie-2017-de-sjm/

Servicio Jesuita a Migrantes. (2018). Informe CIE 2018: Discriminación de Origen. https://sjme.org/publicacion/informe-cie-2018-discriminacion-de-origen/

SOS Racismo. (2018). Informe CIE 2014-2017. Más allá de la frontera de lo humano. https://sosracismo.eu/informe-cie-aluche-2014-2017/

Vargas, J. (2019, 16 de septiembre). España expulsó a 11.384 extranjeros en 2018, un 22\% más que hace un año. Público. https://www.publico.es/sociedad/expulsionesextranjeros-espana-expulso-11384-extranjeros-2018-22-ano.html

\section{Normativa}

Ley Orgánica 10/1995 (23 de noviembre), del Código Penal. Boletín Oficial del Estado, (281), 33987-34058. https://www.boe.es/buscar/doc.php?id=BOE-A-1995-25444

Circular 6/2014, de la Dirección General de la Policía-Comisaría General de Extranjería y Fronteras (11 de julio de 2014) sobre criterios para solicitar el ingreso de ciudadanos extranjeros en Centros de Internamiento. http://www.migrarconderechos.es/legislationMastertable/legislacion/Circular_DGP _CGEF_06 2014

Constitución Española de 1978 (29 de diciembre). Boletín Oficial del Estado, (311), 29313-29424. https://www.boe.es/buscar/act.php?id=BOE-A-1978-31229

Directiva (UE) 2008/115/CE del Parlamento Europeo y del Consejo (16 de diciembre de 2008), relativa a normas y procedimientos comunes en los Estados miembros para el retorno de los nacionales de terceros países en situación irregular. Diario Oficial de la Unión Europea, L 348/98, pp. 98-107. https://www.boe.es/doue/2008/348/L00098-00107.pdf 
Ley Orgánica 10/1995 (23 de noviembre), del Código Penal. Boletín Oficial del Estado, (281). https://www.boe.es/buscar/act.php?id=BOE-A-1995-25444

Ley Orgánica 14/2003, de 20 de noviembre, de Reforma de la Ley orgánica 4/2000 (1 de enero), sobre derechos y libertades de los extranjeros en España y su integración social, modificada por la Ley Orgánica 8/2000, de 22 de diciembre; de la Ley 7/1985, de 2 de abril, Reguladora de las Bases del Régimen Local; de la Ley 30/1992, de 26 de noviembre, de Régimen Jurídico de las Administraciones Públicas y del Procedimiento Administrativo Común, y de la Ley 3/1991, de 10 de enero, de Competencia Desleal. Boletín Oficial del Estado, (279), 41193-41204. https://boe.es/buscar/doc.php?id=BOE-A-2003-21187

Ley Orgánica 7/1985 (1 de julio), sobre derechos y libertades de los extranjeros en España. Boletín Oficial del Estado, (158), 20824-20829. https://www.boe.es/buscar/doc.php?id=BOE-A-1985-12767

Orden de 1999 (22 de febrero) sobre normas de funcionamiento y régimen interior de los centros de internamiento de extranjeros. Boletín Oficial del Estado, (47), 7681-7688. https://www.boe.es/buscar/doc.php?id=BOE-A-1999-4528

Real Decreto 162/2014 (14 de marzo), por el que se aprueba el reglamento de funcionamiento y régimen interior de los centros de internamiento de extranjeros. Boletín Oficial del Estado, (64), 23236-23264. https://www.boe.es/buscar/doc.php?id=BOE-A-2014-2749

Real Decreto 167/2010, de 19 de febrero, por el que se regula la concesión de una subvención directa a favor de Cruz Roja Española para el desarrollo de programas de asistencia integral en el Centro de Internamiento de Extranjeros de Madrid durante el ejercicio 2010. Boletín Oficial del Estado, (45), 16645-16650. https://www.boe.es/diario boe/txt.php?id=BOE-A-2010-2740

Sentencia número 115/1987. (7 de julio). Boletín Oficial del Estado, 180. http://hj.tribunalconstitucional.es/es-ES/Resolucion/Show/847 